\title{
A model comparison method in digital inspection of railway track wear
}

\author{
P. Chen ${ }^{1,2}$, M. Lauer ${ }^{2}$, P. Wang ${ }^{1} \&$ W. Li $^{1}$ \\ ${ }^{1}$ School of Mechanical Engineering, \\ Southwest Jiaotong University, China \\ ${ }^{2}$ Institute of Measurement and Control Systems, \\ Karlsruhe Institute of Technology, Germany
}

\begin{abstract}
Railway track condition has its importance in relation to the driving safety and transport capacity of railways, and its inspection is a crucial task for railway maintenance. Rail wear directly influences wheel-rail contact and the life of rail tracks, so precise and effective inspection of railway wear is a continuous demand. In this paper, a comparison method between point clouds from a structured lightscanner and CAD models of the rail is proposed for railway track wear measurement. With the segmentation algorithm based on Euclidean clustering and random sample consensus (RANSAC), the wheel-rail and non-wheel-rail contact profile are extracted for the alignment. Comparing the measured data and CAD model, random points sampling from CAD models is conducted to generate enough points for the data alignment. In the coarse registration, the curvature of the rail profile is utilized for the point feature histogram $(\mathrm{PFH})$ generation. In the fine registration, the points on non-wheel-rail contact profiles are utilized in the ICP algorithm. To verify the effectiveness of the model comparison, the analysis from software Geomagic Qualify is referenced. It proves that the model comparison method can provide the fundamental support for railway track wear evaluation.
\end{abstract}

Keywords: railway track wear, structured light measurement, point cloud segmentation, point cloud registration. 


\section{Introduction}

The railway track condition has its importance in relation to driving safety and transport capacity of railways, and its inspection is a crucial task for railway maintenance. Rail wear directly influences wheel-rail contact and life of rail tracks. With the increasing wheel-rail contact area caused by wear, both the energy consumption and riding comfort of the trains become degraded. Periodical inspection of the railway track wear is significant for the decisions of replacing the damaged rails and grinding the rail heads. To acquire the vertical and lateral wear amounts of the rail, the precise and effective measurement of rail profile becomes a continuous demand.

There are a series of geometric parameter measurement solutions for rail inspections. MiniProf BT Rail is a contact tool for rail cross section profile measurement made by Green Wood. It provides a telescopic rod attached to the opposite rail for profiling, and analyzes the wear by Envision software package via calculations and alignments [1]. Compared with contact measurement, MERMEC offers a non-contact track measuring system Laserail. It has dual optical measurement boxes to get full profile parameters [2]. Similarly, ENSCO developed a non-contact optical imaging system to measure transverse rail profile, rail wear and rail cant [3]. With a non-contact laser measurement system, the laser is usually projected as a line on the rail that is captured by the cameras for the detected rail profile in images. The wear amount is calculated by aligning the $2 \mathrm{D}$ profile to some reference models. Marcos Nieto et al fit a 3D rail model to the data from a single camera railways track profile inspection system with a particle filter using the efficient overrelation slice sampling method, which can obtain rail wear measurements robustly [4]. Liu et al. [5] utilizes the centers of arcs on large and small circles segmented from rail profile to register the measured rail profile to a reference profile on $2 \mathrm{D}$ scale.

The projecting plane of a laser generally has 1 to $2 \mathrm{~mm}$ width, and then the laser line image is converted into the rail profile composed by single pixels. For more accurate alignment with the reference model, it requires comparatively continuous $2 \mathrm{D}$ profiles with enough features or dense data in 3D scale. However, large amount laser intersections have to be gathered from images along the rail. Structured light is widely used in industrial $3 \mathrm{D}$ profile inspection, which has high accuracy and efficiency. The pattern of structured light can be point, line, stripe, grid, or more complex coded light [6]. To some extent, laser scanners are also a type of structured light equipment. In order to reduce the number of captured images, multiple light stripes can be projected onto the scene at the same time [7]. As a result, an area of the rail surface with less data gaps is captured in only one image pair which could be reconstructed into a dense point cloud. Shape inspection of objects from real world requires data registration for the alignment between measured data and the referenced shape model. The iterative closest point(ICP) algorithm is a typical method for point cloud registration. Based on a good initial pose estimate, it guarantees convergence into an optimal shape registration [8]. To address the computational complexity problem of ICP, a geometrically-based alignment method is used for feature estimation and selection for point 
correspondence search in finding good initial guesses [9]. For 3D point cloud rail profile alignment, the fitting is still implemented by rotating the rail web curve and by parallel shifting the same side rail base line in $2 \mathrm{D}$ plane although the cross section profile of the rail has been obtained by a 3D scanner [10].

For more accurate and quick railway track wear inspection in 3D scale, a comparison method between point clouds from measurements and CAD models of the rail is proposed. The following of the paper is divided into four parts. First, the working principle and the framework of the digital railway track inspection system based on structured light is introduced. Secondly, the point cloud is analyzed and wheel-rail contact profile and non-wheel-rail contact profiles are segmented. In the next part, random point sampling from CAD models is conducted and the alignment of the two groups of point clouds is implemented. Finally, the conclusion is drawn and work in future is described.

\section{Digital railway track inspection based on structured light}

The 3D structured light inspection is a type of technology for acquiring the surface data by projecting some patterns of light on the measured objects. If the 3D scanner projects light with stripe pattern on the measured surface, these stripes will be modulated by the fluctuations of the object surface and change their shapes. It means that these modulated stripes include the $3 \mathrm{D}$ profile data of the measured surface. As is shown in Figure 1, incident light $\mathrm{P}$ beams onto point $\mathrm{A}$ of the referenced surface, but it will beam onto point $B$ if the measured object exists. It can be observed the end point shifts from A to $\mathrm{C}$, and the distance $\mathrm{AC}$ relates to the information $z=h(x, y)$ which could be regarded as the modulation effect of the surface shape on structured light. With a stereo vision system, the deformed stripes are obtained and demodulated, thus the $3 \mathrm{D}$ profile of the object is measured.

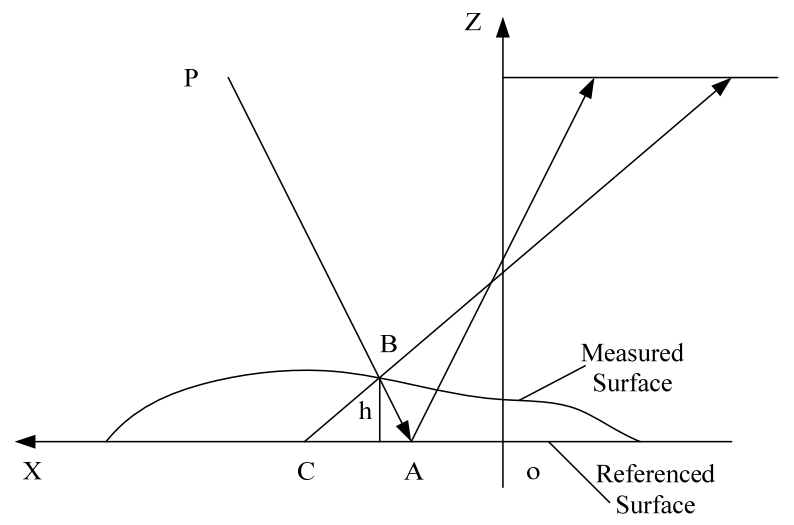

Figure 1: The principle of structured light measurement.

There are two parts in the structured light measurement system as shown in Figure 2. The hardware includes the frame, the CCD or CMOS cameras, the 
structured light projector, the image acquisition card and the computer. The poses of the cameras and projector are adjusted properly so as to cover a certain measured range. In this system, the measured range is $300 \times 400 \mathrm{~mm}$ in the distance of $850 \mathrm{~mm}$. The images of deformed stripes projected on the measured object are input into the computer. The software of the system could extract the encoded stripes in the images, and then calculate the correspondent points according to the camera model and their inner and outer parameters. The 3D point cloud of the surface is generated, and the profile of the object is measured. In this paper, the measured object is the $60 \mathrm{~kg}$ rail in Chinese national standard TB/T2314 in a $500 \mathrm{~mm}$ length section.

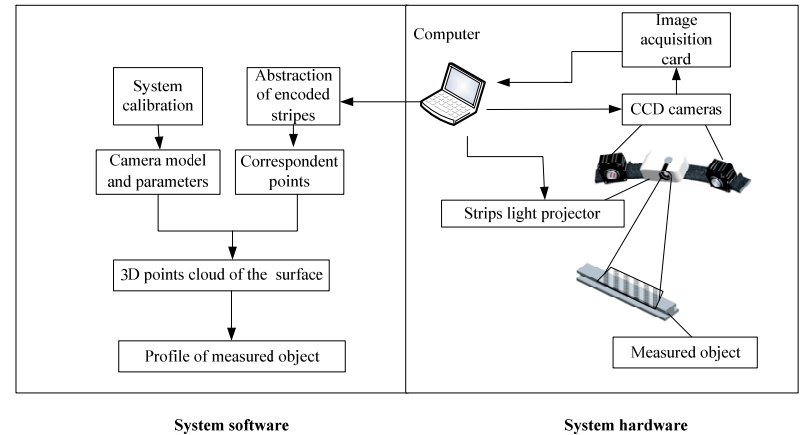

Figure 2: Framework of a structured light measurement system.

\section{Data segmentation of the railway track point cloud}

Railway track is a type of infrastructure with two rails which has its head, web and base parts on the profile. The surface of the rail consists of planar and round corner parts. Parts of the rail head have contact with the wheel of the vehicles, while rail web and rail base are not involved in rail-wheel contact. Because railway track wear occurs on the rail-wheel contact surface of the rail head, this part of the surface could not be used for fine registration in the data alignment. On the contrary, non-wheel-rail contact surfaces with less wear are suitable for this task. According to the structure characteristics of a rail, there are three planes that could be extracted from the point cloud of the rail on a single side data in Figure 3.

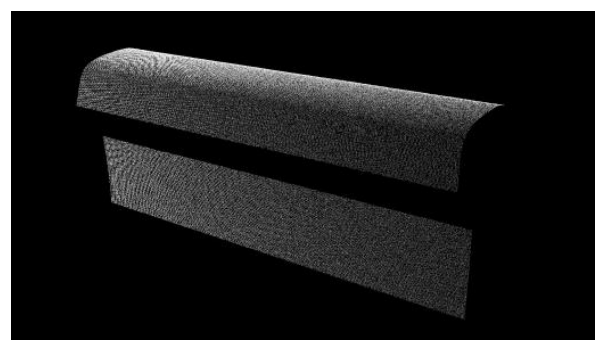

Figure 3: The point cloud of rail on a single side. 
For this data set, a Euclidean clustering method is introduced for extracting cluster data that represent planar surfaces. Within a search radius, the angle deviations between the surface normals of the clustered points are analyzed [11]. A Kd-Tree is created to store the points for searching and extracting, and the segmentation is implemented by the Point Cloud Library(PCL). In Figure 4, the rail head and the rail web are segmented.
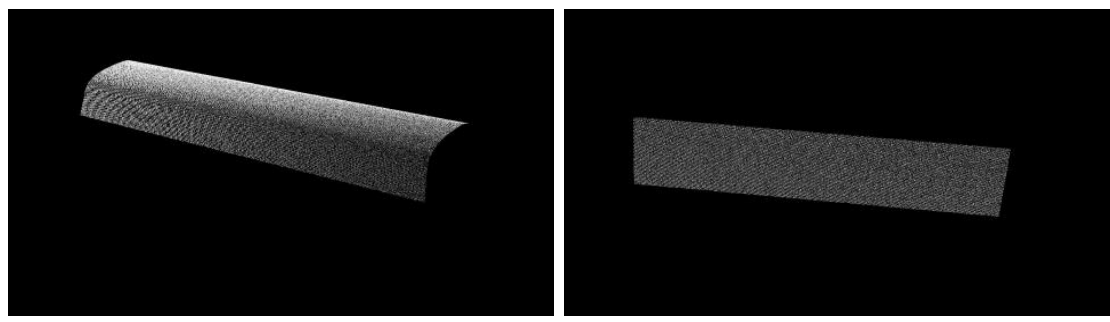

Figure 4: The segmented rail head and the rail web.

The random sample consensus (RANSAC) method can estimate the parameters of a plane model from a point cloud data set. Set a distance threshold, and determine the points on or out of the plane. In the iteration, the largest plane is obtained and removed. The plane extraction is conducted again, and the top surface and side surface of the rail head are segmented as shown in Figure 5.
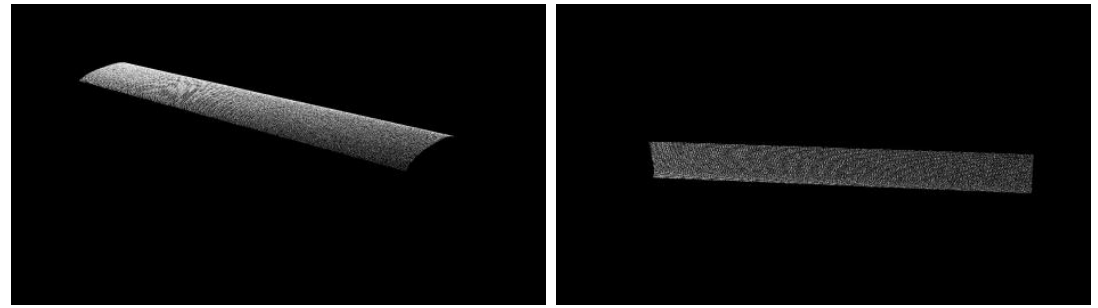

Figure 5: The segmented top surface and side surface of the rail head.

\section{Railway track model comparison}

Model comparison is a crucial step for railway track wear inspection. In traditional railway track wear measurement, the cross section of measurement data and the CAD model are compared, while the comparison between the structured light measurement data and the CAD model is in 3D space. The CAD model is converted into a point cloud, and the comparison is implemented by aligning the measured point cloud to the sampled points from the CAD model. In the alignment, the registration process of point clouds is divided into coarse registration and fine registration by different surfaces segmented from the rail point cloud. For the effectiveness proof of data alignment, the registration results are discussed 
according to the reference analysis from the commercial software Geomagic Qualify.

\subsection{Points sampling from the CAD model}

For the point cloud registration, the point density of the target and reference point clouds should be similar. It can be seen that in the frame style CAD model of the rail in Figure 6, the vertices can only provide very sparse point cloud. Thus, random points sampling from CAD models is carried out to generate enough points for registration.

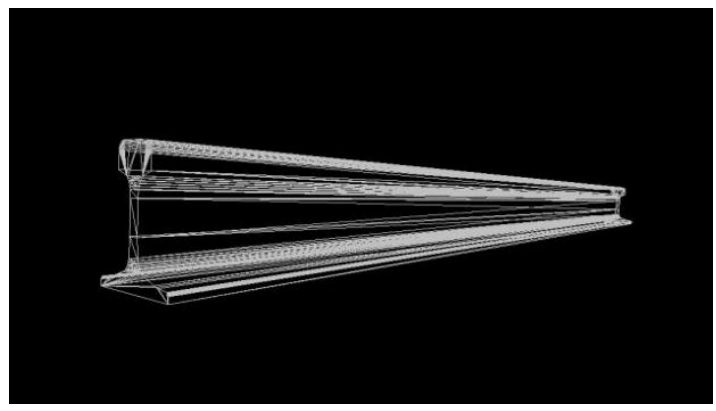

Figure 6: The frame style CAD model of the rail.

As shown in Figure 6, the CAD model is decomposed into a number of triangles. The sampling of points is based on the triangles, and the process is conducted in the steps below.

Step 1. Load the CAD model with triangle meshes and read the coordinates and indexes of vertices, and transform them into point cloud format.

Step 2. Traverse all the triangles, and calculate the areas of all them.

Step 3. Generate the random points in each triangle according to their areas and the required point cloud density.

Step 4. For remaining parts with fractional areas, generate the random points with the same procedure as step 3.

By the random sampling, the CAD model is converted into a point cloud (shown in Figure 7). The density of sampled points can meet the requirements of the model comparison. 


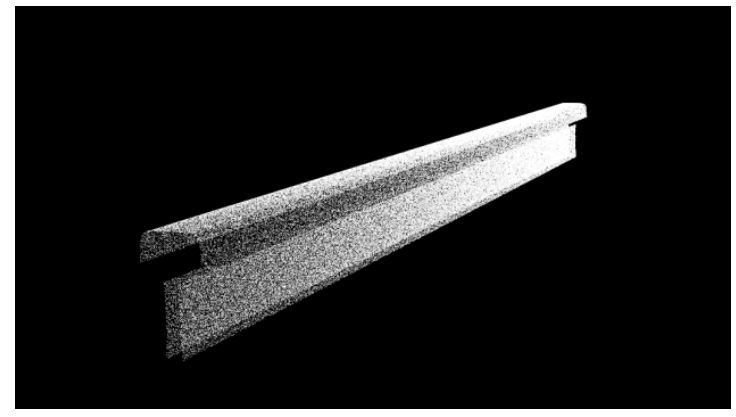

Figure 7: Random sampling result of the CAD model.

\subsection{Alignment method of the sampling points and point cloud from measurement}

A rail is not an object with abundant features that can be extracted for alignment because the cross section of it is almost the same shape along the rail. However, there are two round corners on the top surface of the rail head. They include some geometric features such as curvature, and the surface of the rail head has been segmented in section 3 of this paper. As one of the descriptions of point cloud features, point feature histograms $(\mathrm{PFH})$ are a multi-dimensional histogram with geometric features of query points and their nearest $\mathrm{K}$ neighbors [12]. The PFH descriptor relies on the coordinates and normals of points and their nearest $\mathrm{K}$ neighbors. By computing relationships between the points and their nearest $\mathrm{K}$ neighbors, the point clouds variation on geometric surfaces is presented, which includes the geometric features of the point clouds. The PCL provides the functions for FPH computing, which could be used for the coarse registration of the rail point cloud to the sampling points from its CAD model, and sample consensus initial alignment(SAC-IA) is the algorithm for the coarse alignment. With the support from PCL and the segmentation results of the rail profile, the alignment is implemented as below.

Step 1. Segment the top face of the rail head with round corners as the target for coarse registration.

Step 2. Compute the FPH of the segmented rail head surface and that of the points clouds from CAD model.

Step 3. Align the target point cloud to the reference points from the CAD model in the coarse registration with FPH by SAC-IA algorithm.

Step 4. Conduct the fine registration by the iterative closest point(ICP) algorithm on the side surfaces of rail head and rail web, and align the two groups of point clouds completely.

The ICP algorithm is widely applied in the fine registration, and the function ICP also provided by PCL. There is comparatively less wear on the side surfaces of rail head and rail web, thus it is more suitable to select them as the data set for 
alignment than surfaces from rail-wheel contact profile. The alignment result of the sampling points and the point cloud from measurement is shown as Figure 8. The points with red color are the target from the measured point cloud, and those points with red color from the CAD model are the reference.

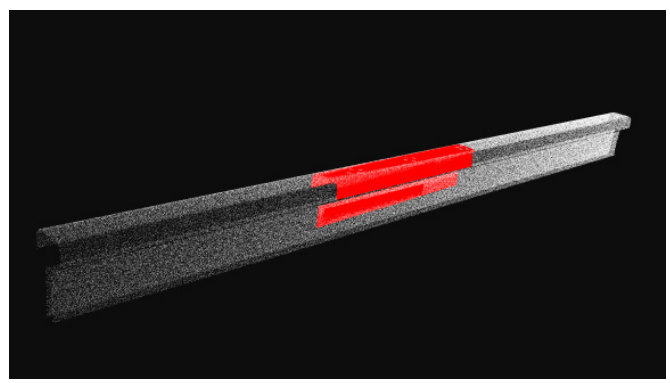

Figure 8: Alignment result of the target and reference model.

\subsection{Discussion of the model comparison results}

The model comparison is implemented by the coarse registration and fine registration for the point clouds alignment. For the fine registration, it is assumed that the measured point clouds are enveloped by the CAD model. The wear amount is the deviation from the CAD model to the measured point cloud, which is acquired by computing the distances of corresponding points in registration. The point cloud of the rail with wear should be enveloped by the CAD model, thus the wear amount is assumed as negative. The distribution of wear amount (in millimeters) by MATLAB is presented in Figure 9.
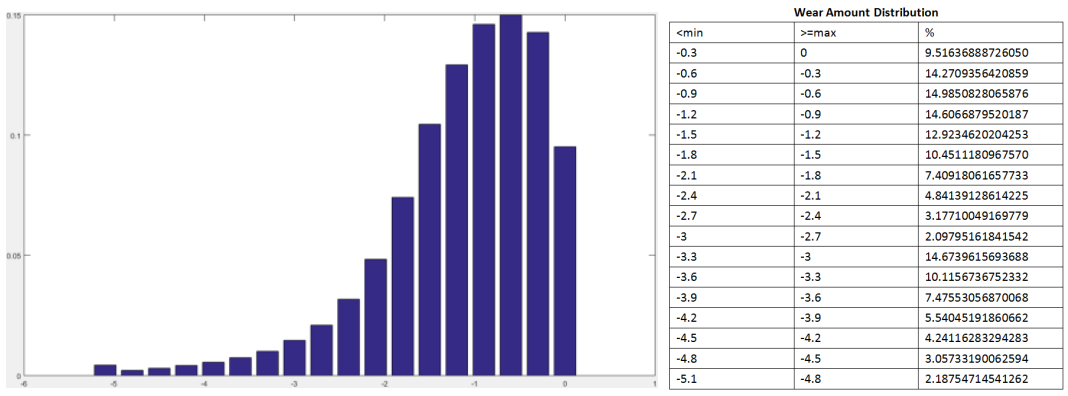

Figure 9: The distribution of wear amount.

To verify the effectiveness of the model comparison, the alignment results between the rail head point cloud and its CAD mesh model are obtained by the commercial software Geomagic Qualify, and the distribution of deviation is also demonstrated in Figure 10. 


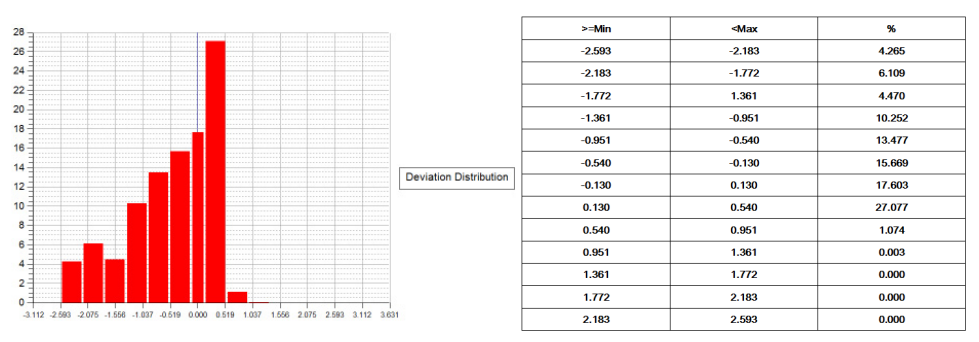

Figure 10: The distribution of deviation in Geomagic Qualify.

In Figure 10, the distribution trend is similar to the counterpart in Figure 9. For the alignment in Geomagic Qualify, the optimal fitting may introduce both negative and positive values. To compare the two groups of distributions, the intervals with the size of 1.5 are taken respectively for analyzing dominant deviations. According to the distribution analysis from the software, the percentage of deviation locates on $[-0.951,0.540]$ is $73.83 \%$. For the statistics result in Figure 9 with the same size interval, the percentage of deviation locates on $[-1.5,0]$ is $66.03 \%$. It should be noted that a part of the positive deviation provided by Geomagic Qualify cannot represented the factual wear amount, so the percentage of deviation in such an interval is smaller than $73.83 \%$. The two distributions reveal similar wear degrees and variations for the same section of the measured rail. It proves that the proposed alignment method has considerable performance consistency with the software Geomagic Qualify, and it can provide the support for railway track wear computation.

\section{Conclusion}

In this paper, a comparison method between point clouds from structured light scanner and CAD models of the rail is proposed for railway track wear measurement. With the segmentation algorithm based on Euclidean clustering and RANSAC, the wheel-rail and non-wheel-rail contact profiles are extracted for the alignment. In the comparison of the measured data and CAD model, random points sampling from CAD models is conducted to generate enough points for the data alignment. In the coarse registration, the curvature of the rail profile is utilized for the PFH generation. In the fine registration, the points on non-wheel-rail contact profiles are utilized in the ICP algorithm. To verify the effectiveness of the model comparison, the analysis from software Geomagic Qualify is referenced. It proves that the model comparison method can provide the fundamental support for railway track wear computation. The work will contribute to the vertical and lateral wearmeasurementin the cross section of the $3 \mathrm{D}$ rail models, and further wear evaluation will be conducted by considering more rail profile parameters in the future. 


\section{Acknowledgements}

The work is supported by the National Natural Science Foundation of China (Grant No. 51305368), Science and Technology Program of Sichuan Province (Grant No. 2013GZX0154) and Fundamental Research Funds for the Central Universities (Grant No. 2682014BR015). The authors also express thanks for the financial support from China Scholarship Council.

\section{References}

[1] GREENWOOD, https://www.greenwood.dk/miniprofbtrail.php

[2] MERMEC, http://www.mermecgroup.com/inspection-technology/trackmeasurement/185/1/track-geometry.php

[3] ENSCO, http://www.ensco.com/products-services/rail-technologies/trackinspection-systems/rail-surface-wear-condition.htm

[4] Nieto, M., Cortés, A., Barandiaran, J., Otaegui, O.\& Etxabe, I., Single Camera Railways Track Profile Inspection Using a Slice Sampling-Based Particle Filter. Computer Vision, Imaging and Computer Graphics. Theory and Application. Springer Berlin Heidelberg, pp. 326-339, 2012.

[5] Liu, Z., Sun, J., Wang, H.\& Zhang, G., Simple and fast rail wear measurement method based on structured light. Optics and Lasers in Engineering, 49(11), pp. 1343-1351, 2011.

[6] Šagi, G., Lulić, Z.\& Mahalec, I., Reverse Engineering. Concurrent Engineering in the 21st Century. Springer International Publishing, pp. 319$353,2015$.

[7] Ribo, M., \& Brandner, M., State of the art on vision-based structured light systems for 3D measurements. IEEE Robotic Sensors: Robotic and Sensor Environments, 2005. International Workshop on, pp. 2-6, 2005.

[8] Jiang, J., Cheng, J.\& Chen, X., Registration for 3-D point cloud using angular-invariant feature. Neurocomputing, 72(16), pp. 3839-3844, 2009.

[9] Rusu, R. B., Blodow, N.\& Beetz, M., Fast point feature histograms (FPFH) for $3 \mathrm{D}$ registration. IEEE International Conference on Robotics and Automation, pp. 3212-3217, 2009.

[10] Zhou, Y., Zhang, J., Jiang, J.\& Yu, M., Study and Implementation on Rail Profile Alignment Algorithm Based on 3D Scanning Data. 15th COTA International Conference of Transportation Professionals, pp. 1942$1951,2015$.

[11] Ali, H., \& Figueroa, N., Segmentation and Pose Estimation of Planar Metallic Objects. IEEE Ninth Conference on Computer and Robot Vision, pp. 376-382, 2012.

[12] Rusu, R. B., Semantic 3D object maps for everyday manipulation in human living environments. KI-Künstliche Intelligenz, 24(4), pp. 345-348, 2010. 